\title{
Rationalising the relation between adlayer structure and observed kinetics in catalysis
}

Michail Stamatakis ${ }^{*}{ }^{\dagger}$ and Simone Piccinin $*$

'Department of Chemical Engineering, University College London, Torrington Place, London WC1E 7JE, United Kingdom

${ }^{\ddagger}$ CNR-IOM DEMOCRITOS c/o SISSA, Via Bonomea 265, 34136 Trieste, Italy

*Corresponding author. e-mail: m.stamatakis@ucl.ac.uk 


\section{Abstract}

Relating the kinetic behaviour of catalytic reactions with adsorbate overlayer structure is a long-standing challenge in catalysis. Even for simple systems such as CO oxidation on $\operatorname{Pd}(111)$, recent studies have observed rich behaviour. In particular, titration experiments by Kondoh and coworkers on this system (J. Chem. Phys. 2006, 124, 224712), demonstrated first-order reaction kinetics with respect to oxygen coverage at $190 \mathrm{~K}$. Yet, the observed kinetics appeared as half-order at $320 \mathrm{~K}$, a phenomenon that was attributed to island formation. We apply a theoretical approach to rationalise these observations, using firstprinciples kinetic Monte Carlo simulations. We employ an analysis that decomposes the overall order into two contributions: one pertaining to the adlayer structure and one quantifying coverage effects on the elementary events' rates. It is shown that at both temperatures the layer appears as almost well-mixed, whereas coverage effects due to adsorbate lateral interactions result in the distinct reaction orders.

\section{Keywords}

Catalysis, $\mathrm{CO}$ oxidation, titration, kinetic Monte Carlo, reaction order, island formation

\section{Background}

Investigating the structure of adsorbate layers on catalytic surfaces and understanding how it affects catalytic activity has attracted and continues to attract a lot of attention from surface scientists and catalysis experts alike. Experimental observables, such as the reaction orders with respect to adsorbates' coverage, a key ingredient in understanding the kinetics of a catalytic system, are typically interpreted in terms of the ordering/geometry of adsorbates' adlayers in Langmuir-Hinshelwood type of reactions. A system with rich kinetics for which this interpretation has been used to explain the change of reaction order with temperature is CO oxidation on $\operatorname{Pd}(111)$.

Early experiments on this catalytic system by Ertl and co-workers ${ }^{1-2}$ as well as Matsushima and co-workers, ${ }^{3}$ provided evidence for a Langmuir-Hinshelwood type of mechanism, and highlighted that adsorption and reaction rate constants are complicated functions of adsorbate coverage. Spectroscopic observations (LEEDS) suggested that, depending on the coverages of $\mathrm{CO}$ and $\mathrm{O}$, different ordered phases of $\mathrm{O}$ emerge, in particular $p(2 \times 2),(\sqrt{3} \times \sqrt{3}) \mathrm{R} 30^{\circ}$ domains and mixed CO-O structures with $(2 \times 1)$ periodicity, as CO coverage increases. ${ }^{3}$ It has been suggested that these phases exhibit different reactivity, with the oxygen $p(2 \times 2)$ being unreactive towards $\mathrm{CO}$ oxidation, and the $(\sqrt{3} \times \sqrt{3}) \mathrm{R} 30^{\circ}$ and $(2 \times 1)$ being active. More recently, Méndez et al. ${ }^{4}$ reported that the $(2 \times 1)$ phase consists of pure oxygen, a statement supported by Green's functions-based STM simulations, contrary to previous experimental work. Seitsonen et al..$^{5}$ have argued that the $\mathrm{O}(\sqrt{3} \times \sqrt{3}) \mathrm{R} 30^{\circ}$ phase is also pure on the basis of DFT calculations of structural parameters of the adlayer and a calculated positive energy of $\mathrm{CO}+\mathrm{O}$ phase mixing, indicating the mixed phase would separate. 
More recently, detailed studies by Kondoh and coworkers ${ }^{6}$ attributed changes in experimental observables to qualitative changes in the structure of the adlayer. A titration protocol was employed in these studies, whereby an O-precovered $\mathrm{Pt}(111)$ surface was exposed to a CO atmosphere and the apparent kinetic parameters were measured while $\mathrm{O}$ was being consumed by the $\mathrm{CO}$ oxidation reaction. It was thus shown that the reaction exhibits different orders with respect to oxygen coverage at different temperatures. ${ }^{6}$ At low temperatures of $190 \mathrm{~K}, 1^{\text {st }}$ order kinetics are observed and attributed to oxygen forming $p(2 \times 1)$ adlayers on the surface. On the other hand, at $320 \mathrm{~K}$, the reaction exhibits half-order kinetics with respect to oxygen and the reaction is thought to proceed on the periphery of $(\sqrt{3} \times \sqrt{3}) \mathrm{R} 30^{\circ} \mathrm{O}$ islands that come in contact with adsorbed CO. This assertion is justified by a geometric argument by which the reaction rate is proportional to the length of these peripheries (reactive regions), which scales with the square root of the coverage, hence the observed order of approximately $1 / 2$.

Inspired by these observations, we recently developed a first-principles-based kinetic Monte Carlo (KMC) model of $\mathrm{CO}$ oxidation on $\operatorname{Pd}(111)^{7}$ and investigated the behaviour thereof at the two different temperatures comparing with the experimental data by Kondoh and coworkers. ${ }^{6}$ The model employed a graph-theoretical KMC framework ${ }^{8}$ and incorporated a cluster expansion Hamiltonian to describe the energetic interactions between adsorbed $\mathrm{CO}$ and $\mathrm{O}$ on fcc and hep lattice sites, as well as Brønsted-Evans-Polanyi (BEP) relations to capture coverage effects on the activation energy of elementary events. ${ }^{9}$ All the parameters that enter the KMC simulations were obtained by density functional theory (DFT) calculations utilising the Perdew-Burke-Ernzerhof $(\mathrm{PBE})^{10}$ generalized gradient approximation (more details can be found in Ref. 7). The simulations were shown to reproduce the titration experiments with remarkable fidelity, including the observed apparent activation energies and reaction orders at the high and low temperature regimes. ${ }^{7}$

There are still, however, several open questions on this deceptively simple system. Why does one observe approximately first-order kinetics at the low temperature $(190 \mathrm{~K})$ versus halforder at the high temperature $(320 \mathrm{~K})$ ? Is the simple geometric argument of island formation versus well-mixed adlayer enough to explain the observed orders? If so, why would island formation be favoured at the high temperature whereby entropy would tend to disperse any clusters? What would then be the driving force behind island formation? On the other hand, could it be that energetic interactions (repulsions/attractions between adsorbates) have an effect on the observed kinetics, and if so, how could this effect be quantified?

In this work we introduce a decomposition scheme to disentangle energetic (coverage) effects versus adlayer structure effects on the apparent reaction orders. While the commonly employed argument to explain reaction orders makes reference to the structure of the adlayer, we show that coverage effects (stemming from adsorbate-adsorbate lateral interactions) have a decisive influence on the observed kinetics.

\section{Extended pure 0 islands: transiently observed but unlikely persistent}

Excluding the possibility of reconstruction on the catalytic surface (which was not observed in the experiment by Kondoh and co-workers), ${ }^{6}$ one would expect to observe extended pure- 
component domains (islands) on the catalytic surface as a result of any of the following driving forces: (1) Attractive interactions between same adsorbates O-O and/or CO-CO. (2) Repulsive interactions between all adsorbates, but with disparate magnitudes between same versus different adsorbates; in particular, repulsive interactions that are much more repulsive between different species (CO-O) than same ones (CO-CO, O-O) could also result in O-rich and CO-rich domains on the surface. (3) Slow diffusion. Our ab initio calculations and KMC simulations support none of these three hypotheses. The lateral interactions between $\mathrm{CO}-\mathrm{CO}$, $\mathrm{O}-\mathrm{O}$ and $\mathrm{CO}-\mathrm{O}$ species are predominantly repulsive with energetic contributions that are similar in magnitude (CO-CO: $0.28 \mathrm{eV}$; O-O: $0.23 \mathrm{eV}$; CO-O: $0.22 \mathrm{eV}$ ), ${ }^{7}$ therefore a thermodynamic driver towards island formation is unlikely. Furthermore, diffusion barriers are 0.53 and $0.26 \mathrm{eV}$ for $\mathrm{O}$ and $\mathrm{CO}$, as opposed to 1.13 and $1.08 \mathrm{eV}$ for $\mathrm{CO}$ oxidation on fcc and hcp sites, ${ }^{7}$ therefore diffusional hops are in principle the fastest processes in this system. Yet, due to the crowded conditions on the catalytic surface, a truly well-mixed behaviour may not be achievable in the beginning of the experiment. In fact, during the random impingement of $\mathrm{CO}$ molecules at the early stages of the experiment, $(\sqrt{3} \times \sqrt{3}) \mathrm{R} 30^{\circ}$ clusters are formed which, however, subsequently intermix with $\mathrm{CO}$ and react, giving away $\mathrm{CO}_{2}$ in the gas phase (our previous simulations were able to reproduce this behaviour for weakly repulsive or mildly attractive $\mathrm{O}-\mathrm{O}$ second nearest neighbour interactions). ${ }^{7}$ Thus, at the low coverages where the experimental measurements of half order kinetics were performed, one would expect almost well-mixed disordered adlayers rather than segregated $\mathrm{O}$ and $\mathrm{CO}$ domains.

\section{Rationalising the experimentally observed reaction orders}

If indeed there is no separation of $\mathrm{O}$ and $\mathrm{CO}$ into distinct domains, the question that arises is how the half order reaction at $320 \mathrm{~K}$ can be explained. To this end, we employed a type of analysis that can decompose the observed reaction order into two contributions, the first pertaining to the structure of the adsorbate overlayer and the second pertaining to the effect of lateral interactions on the kinetic rate constant. This approach is inspired by previous work by Schneider and co-workers ${ }^{11-12}$ who introduced similar decomposition schemes for the apparent activation energy and reaction order of the NO oxidation reaction on $\operatorname{Pt}(111)$.

This analysis is based on the fact that the total rate of an elementary reaction over the lattice can be expressed as a sum of the individual lattice process rates. The sum is taken over all instances of reactive configurations (reaction patterns detected on the lattice):

$$
\text { Rate }=\sum_{\mathrm{i}=1}^{\mathrm{N}_{\text {patt }}} \mathrm{A}_{\mathrm{i}} \cdot \exp \left(-\frac{\mathrm{E}_{\mathrm{a}, 0}+\Delta \mathrm{E}_{\mathrm{a}, \mathrm{i}}^{\mathrm{cov}}}{\mathrm{k}_{\mathrm{B}} \cdot \mathrm{T}}\right)=\sum_{\mathrm{i}=1}^{\mathrm{N}_{\text {patt }}} \mathrm{k}_{\mathrm{i}}
$$

where $\mathrm{N}_{\text {patt }}$ is the number of reaction patterns/instances detected (in our case CO-O reactive pairs), $A_{i}$ the pre-exponential for pattern $i, E_{a, 0}$ the activation energy at the zero coverage limit. Both these kinetic parameters were obtained from DFT calculations as discussed in detail in Ref. 7. Moreover, $\Delta \mathrm{E}_{\mathrm{a}, \mathrm{i}}^{\mathrm{cov}}$ is the contribution to the activation energy arising from coverage effects. The latter is modelled by a BEP relation, such that: 


$$
\Delta \mathrm{E}_{\mathrm{a}, \mathrm{i}}^{\mathrm{cov}}=\omega \cdot\left(\Delta \mathrm{E}_{\mathrm{rxn}, \mathrm{i}}-\Delta \mathrm{E}_{\mathrm{rxn}, 0}\right)
$$

where $\omega$ is a parameter referred to as the proximity factor and ranges between 0 and $1,9,13$ $\Delta \mathrm{E}_{\mathrm{rxn}, \mathrm{i}}$ is the reaction energy under the influence of coverage effects for pattern $\mathrm{i}$, and $\Delta \mathrm{E}_{\mathrm{rxn}, 0}$ is the reaction energy at the zero coverage limit (in the absence of coverage effects). We have previously developed two such BEP relations for $\mathrm{CO}$ oxidation events, one for an event where $\mathrm{CO}$ and $\mathrm{O}$ react from fcc sites, and a second one for both adsorbates reacting from hcp sites. The zero-coverage reaction energies, $\Delta \mathrm{E}_{\mathrm{rxn}, 0}$, were obtained from DFT, as well as the $\omega$-factor for the fcc-related event. For the oxidation event from hcp sites, the same $\omega$-factor as for the fcc sites was used. Since CO oxidation from fcc sites vastly dominates the observed reaction rate, a highly accurate value for $\omega$ on hcp sites is not needed. For the oxidation event from fcc sites we further showed that the BEP developed captures the activation energies within an acceptable error of $0.1 \mathrm{eV}$. $^{7}$

We note that the pre-exponential is assumed to be independent of the environment of the reaction, since the vibrational frequencies of adsorbates are assumed to be unaffected by the presence of spectators. ${ }^{14}$ The rate computed above is an extensive quantity and scales with the number of lattice sites. One can define an instantaneous reaction rate constant averaged over the whole lattice:

$$
\overline{\mathrm{k}}=\frac{1}{\mathrm{~N}_{\text {patt }}} \cdot \sum_{\mathrm{i}=1}^{\mathrm{N}_{\text {patt }}} \mathrm{k}_{\mathrm{i}}
$$

and write the $\log$ of the rate from equation (1) as:

$$
\log (\text { Rate })=\log \left(\mathrm{N}_{\text {patt }}\right)+\log (\overline{\mathrm{k}})
$$

Equation (4) breaks the rate into two contributions: the first term pertains to the number of reactive configurations on the lattice and the second one to the average (energetic) coverage effects felt by the reacting molecules. From equation (1) this second term can also be written as:

$$
\overline{\mathrm{k}}=\mathrm{A} \cdot \exp \left(-\frac{\mathrm{E}_{\mathrm{a}, 0}}{\mathrm{k}_{\mathrm{B}} \cdot \mathrm{T}}\right) \overline{\exp \left(-\frac{\Delta \mathrm{E}_{\mathrm{a}}^{\mathrm{cov}}}{\mathrm{k}_{\mathrm{B}} \cdot \mathrm{T}}\right)}
$$

For a macroscopically homogeneous system, $\mathrm{N}_{\text {patt }}$ and $\overline{\mathrm{k}}$ should be solely functions of coverage; note that this does not necessarily mean that the system is well-mixed. For instance, $\mathrm{N}_{\text {patt }}$ can be computed for the case where reactants are well-mixed or for situations in which there is clustering into islands. $\mathrm{N}_{\text {patt }}$ normalised with respect to the number of sites $\mathrm{N}_{\mathrm{L}}$ will exhibit different scaling with respect to a reactant's coverage in these two different cases. Thus, this decomposition could help identify the contributions of the two components, structure versus energetics, on the reaction order.

Since we are interested in the order with respect to the coverage of oxygen we write: 


$$
\text { Rate }=\mathrm{c} \cdot \mathrm{N}_{\mathrm{L}} \cdot \Theta_{\mathrm{O}}{ }^{\mathrm{n}}
$$

where $\mathrm{c}$ is a constant, $\mathrm{N}_{\mathrm{L}}$ is the number of sites on the lattice (to make the conversion between intensive and extensive rate), and $\mathrm{n}$ is the order of the reaction with respect to oxygen. From equations (4) and (6):

$$
\log \left(\mathrm{N}_{\mathrm{patt}}\right)+\log (\overline{\mathrm{k}})=\log \left(\mathrm{c} \cdot \mathrm{N}_{\mathrm{L}}\right)+\mathrm{n} \cdot \log \left(\Theta_{\mathrm{O}}\right)
$$

The slopes of the logarithms of $\mathrm{N}_{\text {patt }}$ and $\overline{\mathrm{k}}$ with respect to the logarithms of the coverage should therefore sum up to the order of the reaction (n), thereby quantifying the relative contribution of reactive configurations' density (which relates to the adsorbates' location) versus energetics. Note that in our simulations of the titration experiment, changes in the coverage result from the depletion of $\mathrm{O}$ as the reaction proceeds (transient conditions). However, the analysis scheme is general, and could also be applied to steady state conditions. In this case, simulations with partial pressures of a reactant in the feed can result in different steady state coverages, and equation (7) can subsequently be applied.

To validate our analysis scheme, we first applied it to a prototype CO oxidation type of system in which no diffusion takes place and the kinetic constants are independent of coverage. In this system, well-mixed versus segregated behaviour was reproduced by appropriate choice of the initial conditions. The simulation setup and results are shown in the Supporting Information and confirm the intuitive expectation of half-order kinetics for the segregated case and first-order kinetics for the well-mixed case, an effect stemming solely from the adlayer structure.

In the real system however, coverage effects are prominent; therefore we applied the analysis scheme in discussion to KMC trajectories of the titration experiment by Kondoh and coworkers. ${ }^{6}$ We used our previously developed $\operatorname{model}^{7}$ on a larger lattice $(384 \times 192)$ since calculating the average rate constant by equation (3) requires a fairly large number of samples due to the disparity of the values of $\mathrm{k}_{\mathrm{i}}$ that stems from the exponential dependence thereof on the activation energy. To further reduce the noise in the results, we replicated each simulation 10 times using a different random number sequence. In our analysis, we focused on the elementary process of $\mathrm{CO}$ oxidation on fcc sites, since this process had the dominant contribution on the overall rate.

For temperature equal to $190 \mathrm{~K}$ the experiment showed an order of $0.93 \pm 0.03{ }^{6}$ The simulation results at this temperature are shown in Figure 1c, d. We observe that after an initial induction period, the slopes of $\log \left(\mathrm{N}_{\text {patt }}\right)$ and $\log (\overline{\mathrm{k}})$ become almost constant. This will be referred to as the regime of temporal invariance; even though the system never reaches a steady state, the apparent reaction orders are indeed a well-defined observable in this regime. The slopes of $\log \left(\mathrm{N}_{\text {patt }}\right)$ and $\log (\overline{\mathrm{k}})$ versus $\log \left(\Theta_{\mathrm{O}}\right)$ were thus computed for sufficiently low oxygen coverages, after the system reaches a regime of temporal invariance. It is interesting to observe that both the influence of energetics on the average rate and the effect of the adlayer structure result in positive contributions of 0.30 and 0.76 , respectively, to 

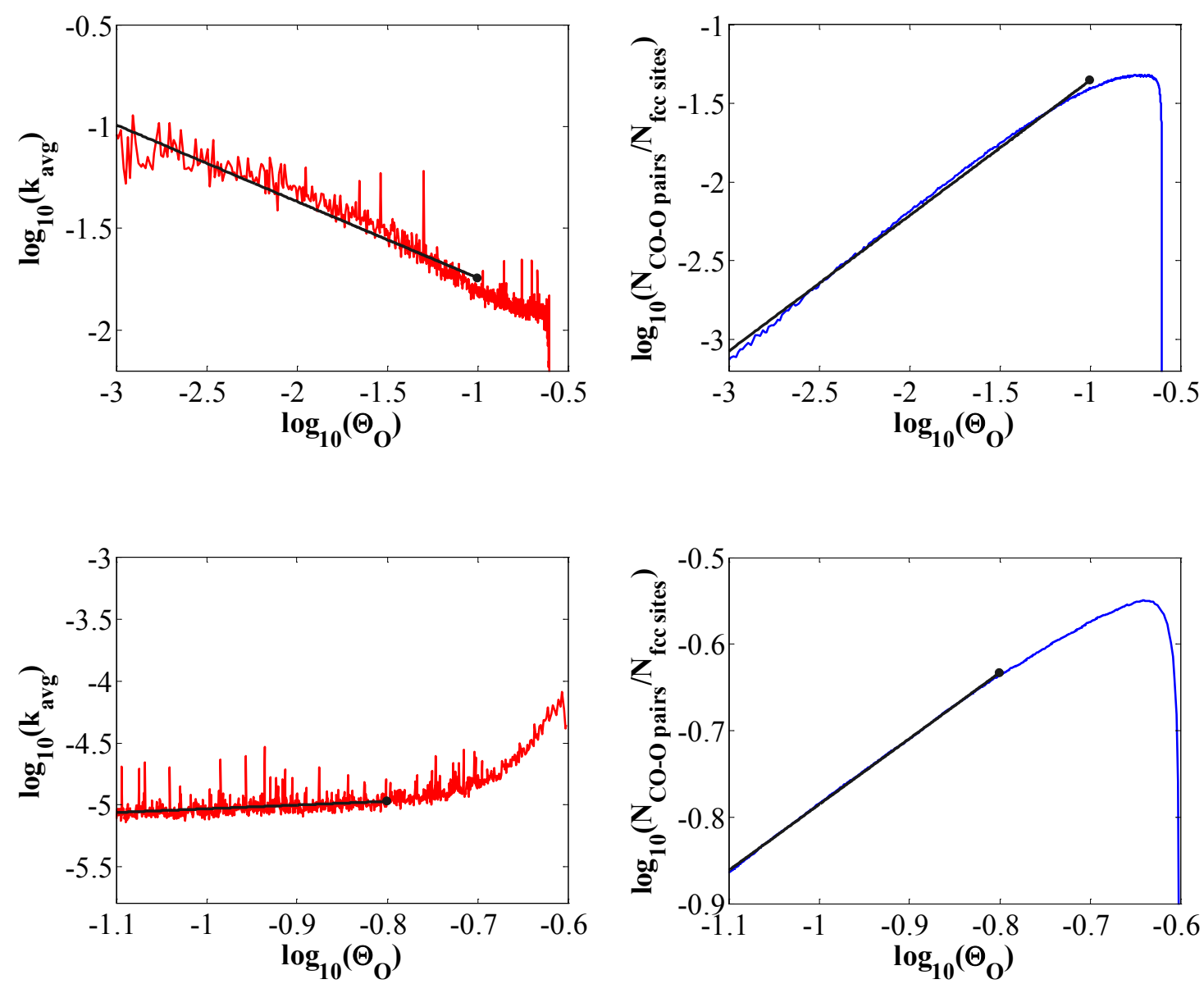

Figure 1: Panel (a): the red trajectory shows the logarithm of $\overline{\mathbf{k}}$ computed via equation (3) with respect to the logarithm of the oxygen coverage at $320 \mathrm{~K}$. The thick black line was obtained from linear fitting of the data at the low oxygen coverage regime, in particular for $\log \left(\Theta_{0}\right) \leq-1.0$ and has a slope of $-\mathbf{0 . 3 8}$. Panel (b): the logarithm of the normalised number of reactive patterns with respect to the logarithm of the oxygen coverage at $320 \mathrm{~K}$. The thick black line was obtained from linear fitting as in panel (a) and has a slope of 0.86 . The sum of the two slopes $(a, b)$ is 0.48 . The choice of the range of fitting was made as follows: by inspecting the coverage-dependence of the pattern density, a linear regime was identified for coverages smaller than $0.1 \mathrm{ML}$. Panels (c) and (d): as in panels (a) and (b) for $T=190 \mathrm{~K}$. The linear fits in this temperature were similarly obtained for $\log \left(\Theta_{0}\right) \leq-\mathbf{0 . 8}$. The slopes for these fits are 0.30 (c) and 0.76 (d) summing up to 1.06. All graphs are averages computed from an ensemble of $10 \mathrm{KMC}$ runs.

the total reaction order. Thus, first order kinetics is observed in this temperature. On the other hand, at $320 \mathrm{~K}$ (panels $\mathrm{a}, \mathrm{b}$ ), the influence of energetics on the average rate result in a negative contribution to the overall order, -0.38 , which added to the 0.86 contribution due to the adlayer structure, eventually yields the half order that is observed under these conditions (experimental value: $0.57 \pm 0.03)^{6}$

Notably, the contributions of adlayer structure to the observed orders are close to unity at both temperatures (panels b, d), suggesting that the $\mathrm{CO}$ and $\mathrm{O}$ adsorbates behave almost in a way that "everyone meets everyone". This can also be verified by visual inspection of the surface configurations, in which both adsorbate types are seen to be located randomly on the lattice, and contradicts the presumption that island formation leads to the observed half order. Instead, we find that the combined effects of (i) lateral interactions on the rates of elementary 
events and (ii) adlayer structure on the density of reactive configurations on the lattice, give rise to the observed kinetic behaviour.

It is also interesting to note that $\log (\overline{\mathrm{k}})$ ranges between higher values for the higher temperature of $320 \mathrm{~K}$ (Figure 1a) compared to the lower temperature of $190 \mathrm{~K}$ (Figure 1b). This behaviour is expected from the Arrhenius relation (see for instance equation 1), which captures the temperature dependence of the elementary event's rate constant. Moreover, the logarithm of the density of patterns exhibits higher values at the low temperature (Figure 1, panel $\mathrm{d}$ versus $\mathrm{b}$ ). This is also in line with intuition; at low temperatures, $\mathrm{CO}$ reaches higher coverages $^{7}$ and thus more $\mathrm{CO}-\mathrm{O}$ pairs can be found on the surface.

\section{The non-trivial behaviour of the average rate constant}

The markedly different behaviour of $\overline{\mathrm{k}}$ in the two different experimental temperatures (190 $\mathrm{K}$ and $320 \mathrm{~K}$ ) is intriguing indeed. To elucidate the origins thereof, we further investigated the relation between elementary $\mathrm{CO}$ oxidation event rates and reaction environment. We note that the repulsive interactions between spectator adsorbates and the reactive $\mathrm{CO}$ and $\mathrm{O}$ destabilise the initial state and consequently the transition state, in line with the BEP relation for the activation energy. ${ }^{9}$ Therefore the initial energies in the absence and in the presence of spectators can be written as follows:

$$
\begin{aligned}
& \mathrm{E}_{\mathrm{rxn}, 0}^{\mathrm{ini}}=\mathrm{E}_{\mathrm{CO}}+\mathrm{E}_{\mathrm{O}}+\mathrm{E}_{\mathrm{CO}-\mathrm{O}} \\
& \mathrm{E}_{\mathrm{rxn}, \mathrm{i}}^{\mathrm{ini}}=\mathrm{E}_{\mathrm{CO}}+\mathrm{E}_{\mathrm{O}}+\mathrm{E}_{\mathrm{CO}-\mathrm{O}}+\mathrm{E}_{\mathrm{CO}-\mathrm{O}, \mathrm{i}}^{\mathrm{CO} \text { cov }}+\mathrm{E}_{\mathrm{CO}-\mathrm{O}, \mathrm{i}}^{\mathrm{O} \text { cov }}
\end{aligned}
$$

where $\mathrm{E}_{\mathrm{CO}}, \mathrm{E}_{\mathrm{O}}$ and $\mathrm{E}_{\mathrm{CO}-\mathrm{O}}$ refer to the reactive $\mathrm{CO}$ and $\mathrm{O}$ pair and $\mathrm{E}_{\mathrm{CO}-\mathrm{O}, \mathrm{i}}^{\mathrm{CO} \text { cov }} \mathrm{E}_{\mathrm{CO}-\mathrm{O}, \mathrm{i}}^{\mathrm{O} \text { cov }}$ refer to the interaction of the reactive pair with the $\mathrm{CO}$ and $\mathrm{O}$ field in the reaction environment. The final state of the reaction is unaffected by the presence of spectators, since $\mathrm{CO}_{2}$ gas no longer interacts with the adlayer. Therefore, the term $\Delta \mathrm{E}_{\mathrm{a}, \mathrm{i}}^{\mathrm{cov}}$ of equation (2) can be expressed as follows:

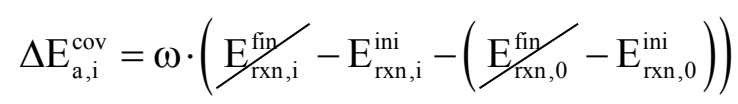

and by use of equations (8):

$$
\Delta \mathrm{E}_{\mathrm{a}, \mathrm{i}}^{\mathrm{cov}}=-\omega \cdot\left(\mathrm{E}_{\mathrm{CO}, \mathrm{O}, \mathrm{i}}^{\mathrm{CO} \text { cov }}+\mathrm{E}_{\mathrm{CO}, \mathrm{O}, \mathrm{i}}^{\mathrm{O} \text { cov }}\right)=-\omega \cdot \mathrm{E}_{\mathrm{cov}, \mathrm{i}}
$$

where the subscript $\mathrm{i}$ means that we refer to a specific reactive pattern (CO-O pair) on the surface, and we have introduced the variable $\mathrm{E}_{\mathrm{cov}, \mathrm{i}}$ to refer to the energetic interactions between the reactive $\mathrm{CO}-\mathrm{O}$ pair in discussion and the $\mathrm{CO} / \mathrm{O}$ spectators. By substituting equation (10) to equation (5) and taking logarithms:

$$
\log (\overline{\mathrm{k}})=\log \left(\mathrm{k}_{0}\right)+\log \left(\overline{\exp \left(\frac{\omega \cdot \mathrm{E}_{\mathrm{cov}}}{\mathrm{k}_{\mathrm{B}} \cdot \mathrm{T}}\right)}\right)
$$


The first term $\log \left(\mathrm{k}_{0}\right)$ on the right hand side is a constant (logarithm of the rate at the zerocoverage limit). The second term is the cumulant generating function for random variable $\mathrm{E}_{\text {cov }}=\mathrm{E}_{\mathrm{CO}, \mathrm{O}}^{\mathrm{CO} \text { cov }}+\mathrm{E}_{\mathrm{CO}, \mathrm{O}}^{\mathrm{O} \text { cov }}$. This function is denoted as $\mathrm{g}(\mathrm{z})$, given as:

$$
g(z)=\log \left(\overline{\exp \left(z \cdot E_{\text {cov }}\right)}\right)=\sum_{j \geq 1} \frac{z^{j}}{j !} \cdot \kappa_{j}
$$

and evaluated at $\mathrm{z}=\omega /\left(\mathrm{k}_{\mathrm{B}} \cdot \mathrm{T}\right)$. If the distribution of $\mathrm{E}_{\mathrm{cov}}$ were known, the cumulants could be evaluated: $\kappa_{1}$ is the mean, $\kappa_{2}$ the variance, $\kappa_{3}$ is the third moment (related to the skewness of the distribution) and higher order cumulants are polynomial expressions of the moments. Since in our case the distribution of $E_{c o v}$ is unknown, we resorted to unbiased estimation of the cumulants from the samples $\mathrm{E}_{\mathrm{cov}, \mathrm{i}}$ generated in the course of the KMC simulation.

We can thus derive an expression for the slope $\Delta \log (\overline{\mathrm{k}}) / \Delta \log \left(\Theta_{\mathrm{O}}\right)$ which is the rate's contribution to the total reaction order (Figure 1):

$$
\frac{\Delta \log (\overline{\mathrm{k}})}{\Delta \log \left(\Theta_{\mathrm{O}}\right)}=\sum_{\mathrm{j} \geq 1} \frac{\mathrm{Z}^{\mathrm{j}}}{\mathrm{j} !} \cdot \frac{\Delta \kappa_{\mathrm{j}}}{\Delta \log \left(\Theta_{\mathrm{O}}\right)}
$$

For $\mathrm{T}=320 \mathrm{~K}$, Figure $2 \mathrm{a}$, displays the evolution of the first 6 terms of the expansion in equation (12), the slopes of which appear in equation (13). Note that the base-10 logarithm is used in the $\mathrm{x}$-axis of this figure for convenient mapping to the actual coverage. The conversion to natural logarithm for calculating the slopes in equation (13) is straightforward, and these slopes are reported in Table 1. We observe that all the cumulants investigated, except from the $1^{\text {st }}$ one (the mean of the energetic interactions), appear to be practically insensitive to the overall oxygen coverage on the lattice. Table 1 confirms this observation and shows that the contributions sum up to -0.39 , which agrees well with the value of -0.38 of Figure 1c.

Since the $1^{\text {st }}$ cumulant-related term dominates as we just discussed, and since the mean of a sum of two random variables is the sum of the means thereof (even if these random variables are not independent), it makes sense to decompose $\kappa_{1}$ into contributions from $\mathrm{CO}$ and $\mathrm{O}$ spectator species (Figure 2b). As expected, the contribution of the interactions for each of the two species follows the same trend as the respective coverage. Yet, the interactions with spectator $\mathrm{CO}$ molecules are slightly larger: the dominant terms are shown in section "Dominant 1NN Energetic Interactions" in Supporting Information, revealing that $\mathrm{CO}-\mathrm{CO}$ repulsions are considerably stronger than the $\mathrm{CO}-$ $\mathrm{O}$ and $\mathrm{O}-\mathrm{O}$ repulsions, whereas $\mathrm{O}-\mathrm{O}$ repulsions are only slightly larger than $\mathrm{CO}-\mathrm{O}$ repulsions. Thus, the replacement of $\mathrm{O}$ adatoms by $\mathrm{CO}$ molecules as the reaction progresses generates new CO-CO pairs, the repulsions in which are enough to compensate for the loss of $\mathrm{O}-\mathrm{O}$

Table 1: Contributions of the first 6 terms in the expansion of equation (13).

\begin{tabular}{rrr}
\hline Temperature & \multicolumn{1}{c}{$320 \mathrm{~K}$} & $190 \mathrm{~K}$ \\
\hline $\mathbf{z} \cdot \Delta \boldsymbol{\kappa}_{\mathbf{1}} / \Delta \log \Theta_{\mathbf{O}}$ & -0.43 & -0.58 \\
$\mathbf{z}^{\mathbf{2}} / \mathbf{2} \cdot \Delta \boldsymbol{\kappa}_{\mathbf{2}} / \Delta \log \Theta_{\mathbf{O}}$ & -0.04 & 2.77 \\
$\mathbf{z}^{\mathbf{3}} / \mathbf{3} ! \cdot \Delta \boldsymbol{\kappa}_{\mathbf{3}} / \Delta \log \Theta_{\mathbf{O}}$ & 0.07 & -2.15 \\
$\mathbf{z}^{\mathbf{4}} / \mathbf{4} ! \cdot \Delta \boldsymbol{\kappa}_{\mathbf{4}} / \Delta \log \Theta_{\mathbf{O}}$ & 0.02 & -4.10 \\
$\mathbf{z}^{\mathbf{5}} / \mathbf{5} ! \cdot \Delta \boldsymbol{\kappa}_{\mathbf{5}} / \Delta \log \Theta_{\mathbf{O}}$ & -0.01 & -0.28 \\
$\mathbf{z}^{\mathbf{6}} / \mathbf{6} \cdot \cdot \Delta \boldsymbol{\kappa}_{\mathbf{6}} / \Delta \log \Theta_{\mathbf{O}}$ & -0.01 & 9.65 \\
\hline Sum & -0.39 & 5.31 \\
\hline
\end{tabular}



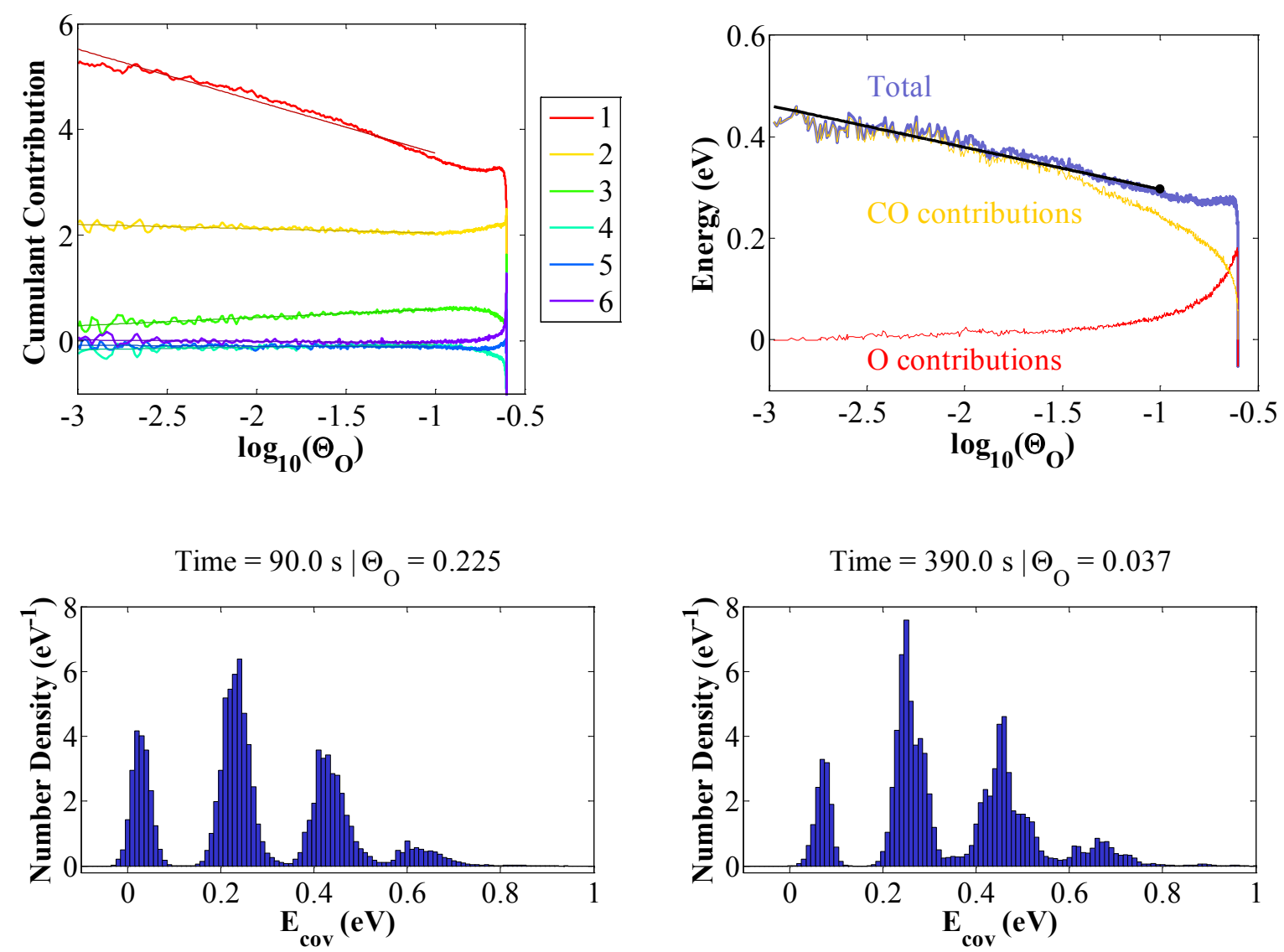

Figure 2: Panel (a): terms of the expansion of $\log (\overline{\mathbf{k}})$ according to equation (12) for $\mathrm{T}=320 \mathrm{~K}$. The terms plotted are of the form $z^{j} / j ! \cdot \kappa_{j}$ for $j=1,2, \ldots, 6$ (as shown in the legend), where $z=\omega /\left(k_{B} \cdot T\right)$ and $\kappa_{j}$ is the $j^{\text {th }}$ cumulant of $\mathrm{E}_{\text {cov }}$. Thin straight lines correspond to linear fits. Calculated from an ensemble of $10 \mathrm{KMC}$ runs. Panel (b): decomposition of the mean of $E_{\text {cov }}\left(\kappa_{1}\right)$ into its constituent contributions of $\mathrm{CO}$ and $\mathrm{O}$. Calculated from a single KMC run. Panels (c, d): The full distributions of $E_{\text {cov }}$ at two different times. Calculated from an ensemble of $10 \mathrm{KMC}$ runs.

interactions, resulting in an overall higher destabilisation of the reactive $\mathrm{CO}-\mathrm{O}$ initial and transition states. This analysis shows that the negative slope of $\log (\overline{\mathrm{k}})$ versus $\log \left(\Theta_{\mathrm{O}}\right)$ can be explained on the basis of destabilisation of initial state induced predominantly by $\mathrm{CO}$ which lowers the barriers and therefore increases the rate.

It is interesting to highlight that, even though for $320 \mathrm{~K}$ the mean of the repulsions dominates the behaviour of the system, the distribution of the repulsions that the reacting CO-O pair "experiences" is rather complicated. In particular, Figure $2 \mathrm{c}, \mathrm{d}$ shows this distribution for two different times (specifically $90 \mathrm{~s}$ and $390 \mathrm{~s}$ ), which are, respectively, an early snapshot at the onset of the regime of temporal invariance, and a later snapshot within that regime. The multimodal appearance of the distribution means that higher cumulants of equation (12) are non-zero; yet, they do not contribute to the slope $\Delta \log (\overline{\mathrm{k}}) / \Delta \log \left(\Theta_{\mathrm{O}}\right)$ because (i) they do not change much with respect to $\log \left(\Theta_{\mathrm{O}}\right)$, as evidenced by the shape of the distribution remaining almost constant, and (ii) they are damped by the term $z^{j} / j$ ! (see section "Higher Order Cumulant Expansion Terms for $\mathrm{T}=320 \mathrm{~K}$ " in Supporting Information). By comparing the distributions of panels (c) and (d) of Figure 2, we still see that as oxygen coverage drops the distribution of $\mathrm{E}_{\mathrm{cov}}$ shifts to higher values, resulting in a larger degree of destabilisation of the 


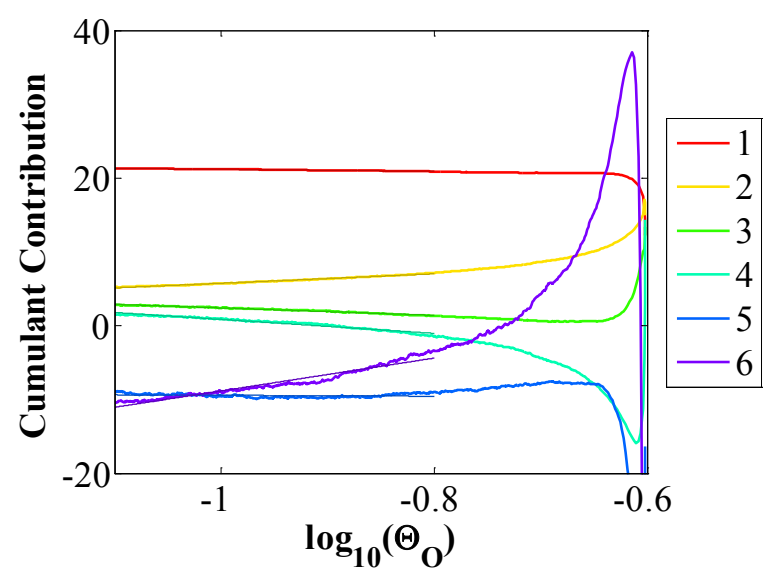

Time $=12000.0 \mathrm{~s} \mid \Theta_{\mathrm{O}}=0.177$

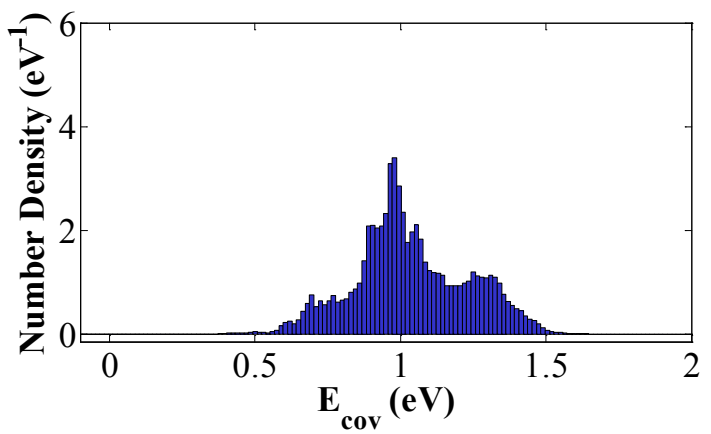

Time $=59700.0 \mathrm{~s} \mid \Theta_{\mathrm{O}}=0.079$

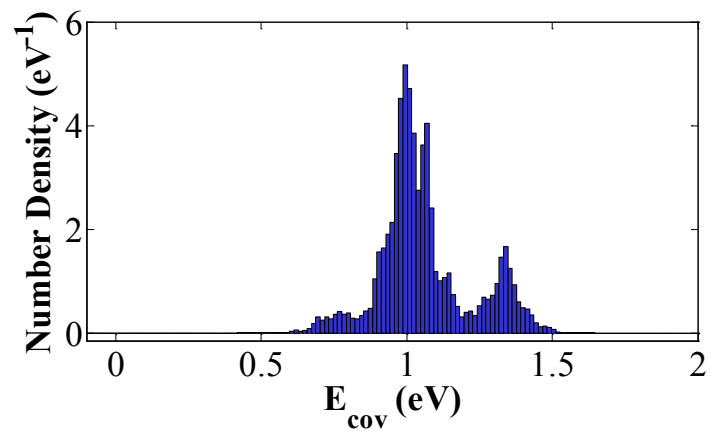

Figure 3: Panel (a): terms of the expansion of $\log (\overline{\mathbf{k}})$ for $\mathrm{T}=190 \mathrm{~K}$ (similar to Figure 2a). Panels (b, c): The full distributions of $E_{\text {cov }}$ at two different times. All plots generated from an ensemble of 10 KMC runs.

initial state and therefore higher instantaneous rates. This effect is in line with the negative slope of $\log (\overline{\mathrm{k}})$ versus $\log \left(\Theta_{\mathrm{O}}\right)$ as discussed above.

The situation is more complicated for $\mathrm{T}=190 \mathrm{~K}$. In fact, for this case, the cumulant expansion (equation 12) is not very helpful as higher order terms (which are difficult to interpret) have a significant contribution (Table 1, Figure 3a). We numerically estimated cumulants up to $\kappa_{10}$ and found that the corresponding term in the expansion is significant. The reason for this behaviour can be sought in the evolution of the distribution of $\mathrm{E}_{\text {cov }}$ (Figure $3 \mathrm{~b}, \mathrm{c})$. Two main features emerge by inspecting the distributions' snapshots of Figure $3 \mathrm{~b}, \mathrm{c}$, which correspond to the onset of and a later stage within the regime of temporal invariance. First, there is a depletion of density in the region of high repulsive interactions, specifically $\mathrm{E}_{\mathrm{cov}}$ in the range of 1.2 to $1.5 \mathrm{eV}$, as time progresses. This region corresponds to spectator arrangements that give rise to significant destabilisation of the initial state due to these interactions and result in lower barriers, as expressed by equations (1) and (10), and thus high instantaneous rates (equation 11). On the other hand, there is an accumulation of density in the region of low repulsive interactions $(1 \mathrm{eV})$ and thus lower rates. These two features result in a net decrease of $\log (\overline{\mathrm{k}})$ as time progresses and oxygen gets depleted. This is in agreement with the observed positive slope of $\log (\overline{\mathrm{k}})$ versus $\log \left(\Theta_{\mathrm{O}}\right)$ in Figure 1c, and at variance with the negative slope observed for $\mathrm{T}=320 \mathrm{~K}$. Hence, even though the average of the distribution does not change much (Figure 3a, red curve), the shape thereof has a marked effect in shaping the behaviour of the system, contrary to the case of $320 \mathrm{~K}$. 


\section{Conclusion}

Even simple catalytic systems such as the $\mathrm{CO}$ oxidation reaction on $\mathrm{Pd}(111)$, can exhibit a remarkably complex behaviour. The rich kinetics demonstrated by the titration experiments of Kondoh and co-worker were initially attributed to the different $\mathrm{O}$ ordered phases having different reactivity, and the active sites of the reaction switching from interstitial sites of $p(2 \times 1)$ domains at $190 \mathrm{~K}$ (first order reaction with respect to $\mathrm{O}$ ), to the periphery of $(\sqrt{3} \times \sqrt{3}) \mathrm{R} 30^{\circ}$ domains at $320 \mathrm{~K}$ (half-order reaction with respect to $\mathrm{O}$ ).

We previously developed a first-principles based model that was able to reproduce this kinetic behaviour with remarkable fidelity. We have now employed analysis schemes that have allowed us to rationalise this behaviour, thereby (i) decomposing the apparent reaction order into contributions that stem from the adlayer structure (geometry) and the adsorbateadsorbate lateral interactions (energetics); (ii) further decomposing the effect of energetics into contributions by the mean-field of spectators but also more complicated descriptors (higher-order cumulants) related to the entire distribution of the energetic interaction contributions to the activation energies. To our knowledge, this latter decomposition has not been considered before, and may hold the key to assessing the quality of mean-field approximations commonly employed in models of catalytic reactions.

Our results suggest that while $(\sqrt{3} \times \sqrt{3}) \mathrm{R} 30^{\circ}$ domains are observed transiently at $320 \mathrm{~K}$, the reaction is not limited to the periphery thereof, rather, the adlayer appears as almost wellmixed at both temperatures $(190 \mathrm{~K}$ and $320 \mathrm{~K})$. The puzzling half order with respect to $\mathrm{O}$ at $320 \mathrm{~K}$ was attributed to the energetic interactions between $\mathrm{CO}-\mathrm{O}$ reactants and $\mathrm{CO}$ spectators that progressively accumulate on the surface. Thus, as $\mathrm{O}$ gets depleted, on the one hand the elementary oxidation event rates increase, due to the stronger destabilisation of the initial and transition states, but on the other hand CO-O reactive pairs are found less frequently on the adlayer. The net effect manifests as half order kinetics. These results challenge the traditional view that half order kinetics merely stem from island formation (segregation of adsorbates) on the surface.

Our simulations at $190 \mathrm{~K}$ and their analysis via cumulant expansions revealed that the entire distribution of spectator energetic interactions is important. As time increases and $\mathrm{O}$ gets depleted, spectator arrangements that destabilise the initial and transition states are becoming less frequent. On the other hand, more stable arrangements emerge and thus the elementary oxidation event rates decrease. This effect, in addition to the less frequently occurring $\mathrm{CO}-\mathrm{O}$ reactive pairs as $\mathrm{O}$ gets depleted, manifests as first order kinetics at this temperature.

We have thus demonstrated the analytical power of first-principles based kinetic modelling in elucidating the complex behaviour of a deceptively simple system. Such approaches that integrate the various sources of complexity encountered in catalytic systems may hold the key to not only understanding puzzling experimental observations, but also engineering optimal catalysts for bespoke applications. 


\section{Acknowledgements}

The authors are grateful to the UCL Research Software Development Team, funded by the Edinburgh Parallel Computing Centre under project eCSE01-001, for the continuing development of KMC software Zacros employed in the computational part of this work. The high performance computing facilities Legion at UCL and Iridis at the University of Southampton within the e-Infrastructure South Centre for Innovation, as well as the associated support services, are also gratefully acknowledged.

\section{Supporting Information.}

(i) Application of the analysis scheme to well-mixed versus segregated model systems. (ii) Dominant $1 \mathrm{NN}$ energetic interaction patterns in the cluster expansion. (iii) Higher order cumulant expansion terms for $\mathrm{T}=320 \mathrm{~K}$. This material is available free of charge via the Internet at http://pubs.acs.org.

\section{References}

(1) Engel, T.; Ertl, G. J. Chem. Phys. 1978, 69, 1267-1281.

(2) Conrad, H.; Ertl, G.; Küppers, J. Surf. Sci. 1978, 76, 323-342.

(3) Matsushima, T.; Asada, H. J. Chem. Phys. 1986, 85, 1658-1668.

(4) Méndez, J.; Kim, S. H.; Cerdá, J.; Wintterlin, J.; Ertl, G. Phys. Rev. B 2005, 71, 0854091-085409-13.

(5) Seitsonen, A. P.; Kim, Y. D.; Schwegmann, S.; Over, H. Surf. Sci. 2000, 468, 176-186.

(6) Nakai, I.; Kondoh, H.; Shimada, T.; Resta, A.; Andersen, J. N.; Ohta, T. J. Chem. Phys. 2006, 124, 224712-1-224712-8.

(7) Piccinin, S.; Stamatakis, M. ACS Catal. 2014, 4, 2143-2152.

(8) Stamatakis, M.; Vlachos, D. G. J. Chem. Phys. 2011, 134, 214115-1-214115-13.

(9) Nielsen, J.; d'Avezac, M.; Hetherington, J.; Stamatakis, M. J. Chem. Phys. 2013, 139, 224706-1-224706-13.

(10) Perdew, J. P.; Burke, K.; Ernzerhof, M. Phys. Rev. Lett. 1996, 77, 3865-3868.

(11) Wu, C.; Schmidt, D. J.; Wolverton, C.; Schneider, W. F. J. Catal. 2012, 286, 88-94.

(12) Bray, J. M.; Schneider, W. F. ACS Catal. 2015, 5, 1087-1099.

(13) Grabow, L. C.; Gokhale, A. A.; Evans, S. T.; Dumesic, J. A.; Mavrikakis, M. J. Phys. Chem. C 2008, 112, 4608-4617.

(14) Schmidt, D. J.; Chen, W.; Wolverton, C.; Schneider, W. F. J. Chem. Theory Comput. 2012, 8, 264-273. 
TOC Graphic

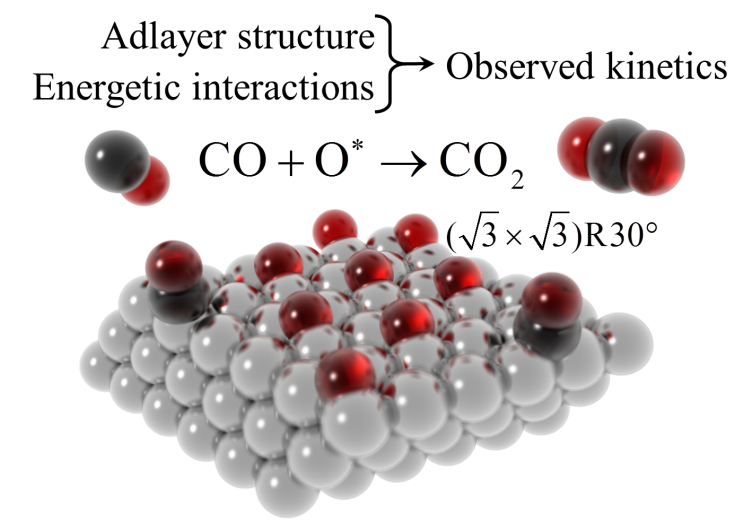

